\title{
Linker stability influences the anti-tumor activity of acetazolamide-drug conjugates for the therapy of renal cell carcinoma
}

\author{
Samuele Cazzamalli ${ }^{\S}$, Alberto Dal Corso ${ }^{\S}$, Dario Neri*
}

Department of Chemistry and Applied Biosciences, Swiss Federal Institute of Technology (ETH Zürich), Vladimir-Prelog-Weg 4, CH-8093 Zürich (Switzerland)

$\S$ ) These authors contributed equally to this work

*) Corresponding Author

Tel: +41-44-6337401

e-mail: neri@pharma.ethz.ch

Running title:

CAIX targeting and linker stability

Keywords:

Drug Release; Tumor Targeting; Small Molecule-Drug Conjugates; Targeted Pro-drugs; Renal Cell Carcinoma; Therapy Studies

\section{Disclosure of potential conflict of interest:}

D.N. is a co-founder and shareholder of Philogen SpA, a company which develops antibody therapeutics and small molecule-drug conjugates

Submitted as Research Article to Journal of Controlled Release 


\begin{abstract}
Small molecule-drug conjugates (SMDCs) are increasingly being considered as an alternative to antibody-drug conjugates (ADCs) for the selective delivery of anticancer agents to the tumor site, sparing normal tissues. Carbonic anhydrase IX (CAIX) is a membrane-bound enzyme, which is over-expressed in the majority of renal cell carcinomas and which can be efficiently targeted in vivo, using charged derivatives of acetazolamide, a small heteroaromatic sulfonamide. Here, we show that SMDC products, obtained by the coupling of acetazolamide with monomethyl auristatin E (MMAE) using dipeptide linkers, display a potent anti-tumoral activity in mice bearing xenografted SKRC-52 renal cell carcinomas. A comparative evaluation of four dipeptides revealed that SMDCs featuring valine-citrulline and valine-alanine linkers exhibited greater serum stability and superior therapeutic activity, compared to the counterparts with valine-lysine or valinearginine linkers. The most active products substantially inhibited tumor growth over a prolonged period of time, in a tumor model for which sunitinib and sorafenib do not display therapeutic activity. However, complete tumor eradication was not possible even after ten intravenous injection. Macroscopic near-infrared imaging procedures confirmed that ligands had not lost the ability to selectively localize at the tumor site at the end of therapy and that the neoplastic masses continued to express CAIX. The findings are of mechanistic and of therapeutic significance, since CAIX is a non-internalizing membrane-associated antigen, which can be considered for targeted drug delivery applications in kidney cancer patients.
\end{abstract}




\section{Introduction}

The majority of low molecular weight drugs, commonly used for cancer chemotherapy, do not preferentially localize to solid tumors in mice $[1,2]$ and patients [3]. This unfavorable pharmacokinetic behavior may lead to undesired toxicity to normal organs and may limit the dose escalation to therapeutically active regimens. In order to improve the therapeutic activity of anticancer chemotherapy, the coupling of cytotoxic agents to monoclonal antibodies has been proposed and experimentally explored, at the preclinical and clinical level [4,5,6,7]. Several monoclonal antibody-drug conjugates (ADCs) have exhibited promising therapeutic results in mouse models of cancer and two products (Adcetris ${ }^{\mathrm{TM}}$ and Kadcyla ${ }^{\mathrm{TM}}$ ) have gained marketing authorization for the treatment of certain malignancies in patients. However, the clinical performance of ADC products is often limited by the slow extravasation of the antibody molecule [8], suboptimal in vivo selectivity [9] and premature drug release $[10,11,12]$, which may contribute to undesired toxicities and limit therapeutic efficacy.

Small molecule-drug conjugates (SMDCs), capable of selective localization at the tumor site, have been proposed as an alternative to ADCs [13]. Small organic ligands, specific to accessible tumor-associated antigens, extravasate and diffuse into tissues more rapidly than antibodies $[14,15]$, These pharmacokinetic characteristics may lead to favorable tumor:blood and tumor:organ ratios at early time points (e.g., few hours) after intravenous administration [16]. Small molecule ligands have been used for the selective in vivo targeting of tumors expressing the folate receptor $[17,18]$, prostate-specific membrane antigen [16,19] and somatostatin receptors [20]. A number of SMDC products are currently being investigated in clinical trials [21]

Carbonic anhydrase IX (CAIX) is a membrane-bound homodimeric enzyme, which is undetectable in most normal adult tissues [22]. CAIX can be found in certain gastro-intestinal structures 
(e.g., stomach, duodenum and gallbladder) [23], albeit in a catalytically-inactive form [24], and in hypoxic tissues [25]. Interestingly, CAIX is also strongly expressed in the majority of kidney cancers, as a result of von Hippel-Lindau mutations and the in vivo ligand-based targeting of this enzyme is more efficient in tumors, compared to normal organs [26]. Moreover, the antigen has been reported to be abundant in a subset of patients with different cancers (i.e., lung, colorectum, stomach, pancreas, breast, cervix, bladder, ovaries, brain, head and neck and oral cavity [27]) with an over-expression at the growing front of the tumor [28].

Even though CAIX has previously been claimed to be an internalizing antigen and has been considered for industrial ADC product development activities [29], our lab has experimentally shown that the protein remains membrane-bound and does not efficiently internalize upon small-ligand binding $[30,31]$.

Acetazolamide is a small heteroaromatic sulfonamide, which binds to various carbonic anhydrases with high affinity. Derivatives of acetazolamide containing multiple charges do not efficiently cross the cell membrane and are restricted for binding to membrane-accessible carbonic anhydrases (i.e., CAIX, but also potentially CAIV and CAXII). We have previously shown that certain acetazolamide derivatives selectively localize to renal cell carcinomas in vivo $[30,32,33]$ and that those ligands can be used for the selective delivery of highly cytotoxic maytansinoids (e.g., DM1) to kidney tumors. Interestingly, the use of disulfide linkers for the coupling of DM1 to acetazolamide allows an efficient and selective drug release at the tumor site, where dying cells release large amounts of glutathione and other reducing agents. Indeed, disulfide linkers have been proposed as selective modules for drugs release also with antibody-drug conjugates $[34,35]$ and with polymer-drug conjugates [36]. 
In this article, we describe the synthesis and characterization of four SMDCs, in which the acetazolamide moiety was coupled to monomethyl auristatin E (MMAE, the payload in Adcetris ${ }^{\mathrm{TM}}$ ) via cleavable linkers, featuring four different dipeptide structures. We observed that valinecitrulline and valine-alanine linkers were more stable in serum, compared to the charged valinelysine and valine-arginine structures. Interestingly, the two most stable SMDCs were also the most therapeutically active products, when tested in mice with xenografted SKRC-52 tumors. These findings are of potential therapeutic significance, as the CAIX targeting agents could be considered for applications in humans. Furthermore, our data indicate that potent therapeutic activity can be achieved in vivo, using non-internalizing ligands and linkers, which had previously been postulated to be processed only in intracellular compartments, by cathepsin B cleavage $[37,38]$. 


\section{Materials and Methods}

$\underline{\text { General remarks }}$

Compound 1 [30] and Fmoc-Lys(Mtt)-PABOH [39] were prepared according to literature procedures and their analytical data were in agreement with those already published. MC-ValCitPABC-MMAE was purchased from Levena Biopharma (No.9 Weidi Road, Qixia District, Nanjing, 210046, China). Maleimidoethyl-IRDye680RD was purchased from LI-COR Biosciences (4647 Superior Street Lincoln, Nebraska USA 68504-5000). The synthetic procedures for the preparation of the ValAla, ValLys, ValArg analogues, of the final SMDCs 2-5 and of the near-infrared dye derivative $\mathbf{6}$ are included in the Supplementary Information, along with NMR/HPLC-MS data and the statistical analysis of the in vivo experiments.

\section{$\underline{\text { General Procedure for Acetazolamide-Drug Conjugation }}$}

Compound 1 (4 eq) was dissolved in phosphate buffered saline (PBS; $50 \mathrm{mM}$ phosphate, $100 \mathrm{mM}$ $\mathrm{NaCl}, \mathrm{pH}$ 7.4) and a solution of MC-linker-PABC-MMAE (1 eq) in DMF was added. The mixtures were stirred at room temperature until completion (monitored by HPLC-MS) and the solvents were removed under vacuum. The conjugates were purified through RP-HPLC and lyophilized. The purity (> 95\%) of the final compounds was detected by analytical HPLC-MS.

\section{Linker Stability Assays}

Compounds 1-4 were incubated at $37{ }^{\circ} \mathrm{C}$ in a shaking incubator as PBS solutions (pH 7.4; 100 $\mu \mathrm{g} / \mathrm{ml})$. Aliquots $(200 \mu \mathrm{l})$ were collected at subsequent time points $(0,15 \mathrm{~min}, 1 \mathrm{~h}, 3 \mathrm{~h}, 6 \mathrm{~h}, 24 \mathrm{~h}$, $96 \mathrm{~h}$ ) and frozen at $-20{ }^{\circ} \mathrm{C}$. The samples were analyzed in triplicate by LC-MS. Peaks relative to the pro-drugs were integrated and area values from samples at different time points were used to 
obtain fractions of intact test compounds. Fraction values were plotted to obtain stability profiles showed in figure 2.

Analogously, test compounds were dissolved in DMSO $(10 \mu \mathrm{l})$, diluted with freshly thawed mouse serum (Invitrogen) $(100 \mu \mathrm{g} / \mathrm{ml})$ and incubated at $37^{\circ} \mathrm{C}$ in a shaking incubator. Aliquots $(100 \mu \mathrm{l})$ were collected at subsequent time points $(0,20 \mathrm{~min}, 1 \mathrm{~h}, 3 \mathrm{~h}, 6 \mathrm{~h}, 24 \mathrm{~h}$ and $48 \mathrm{~h})$ and frozen at $-20^{\circ} \mathrm{C}$. Samples were thawed and diluted with four volumes of $\mathrm{MeOH}(400 \mu \mathrm{l})$. After vigorous vortex agitation for $1 \mathrm{~min}$, the protein precipitate was spun down and $400 \mu \mathrm{l}$ of the supernatant was lyophilized overnight. The resulting solid material was re-dissolved in $200 \mu \mathrm{l}$ of water, filtered and analyzed as described above.

\section{Cell Cultures}

The human renal cell carcinoma cell line SKRC-52 was kindly provided by Professor E. Oosterwijk (Radbound University Nijmegen Medical Centre, Nijmegen, The Netherlands) and tested for CAIX expression by immunofluorescence. No further authentication was performed. SKRC-52 were maintained in RPMI medium (Invitrogen) supplemented with fetal calf serum (10\%, FCS, Invitrogen) and Antibiotic-Antimycotic (1\%, AA, Invitrogen) at $37{ }^{\circ} \mathrm{C}$ and $5 \% \mathrm{CO}_{2}$. For passaging, cells were detached using Trypsin-EDTA $0.05 \%$ (Invitrogen) when reaching $90 \%$ confluence and re-seeded at a dilution of 1:6.

\section{$\underline{\text { In Vitro Cytotoxicity Assay }}$}

SKRC-52 cells were seeded in 96-well plates in RPMI added with 10\% FCS (100 $\mu$ l) at a density of 5000 cells per well and allowed to grow for $24 \mathrm{~h}$. The medium was replaced with medium containing different concentrations of test substance (1:3 dilution steps) and plates were incubated at $37{ }^{\circ} \mathrm{C}$ and $5 \% \mathrm{CO}_{2}$. After $72 \mathrm{~h}$ the medium was replaced by a solution of MTS cell viability dye (20 $\mu 1$, Promega) in $150 \mu \mathrm{l}$ of medium. Plates were incubated for $2 \mathrm{~h}$ under culture conditions and 
the absorbance at $490 \mathrm{~nm}$ measured on a Spectra Max Paradigm multimode plate reader (Molecular Devices). Experiments were performed in triplicate and average cell viability calculated as measured background corrected absorbance divided by the absorbance of untreated control wells. $\mathrm{IC}_{50}$ values were determined by fitting data to the four-parameter logistic equation, using a Prism 6 software (GraphPad Software) for data analysis.

\section{$\underline{\text { Animal Studies }}$}

All animal experiments were conducted in accordance with Swiss animal welfare laws and regulations under the license number 27/2015 granted by the Veterinäramt des Kantons Zürich.

\section{Implantation of Subcutaneous SKRC-52 Tumors}

SKRC-52 cells were grown to $80 \%$ confluence and detached with Trypsin-EDTA $0.05 \%$ (Life Technologies). Cells were washed with Hank's Balanced Salt Solution (HBSS, pH 7.4) once, counted and re-suspended in HBSS to a final concentration of $3.4 \times 10^{7}$ cells $/ \mathrm{ml}$. Aliquots of $5 \times$ $10^{6}$ cells $(150 \mu \mathrm{l}$ of a suspension) were injected subcutaneously in the right flank of female athymic Balb/c nu/nu mice (6-8 weeks of age, Janvier).

\section{Therapy Experiments}

SKRC-52 xenografted tumors were implanted into female Balb/c nu/nu mice (Janvier) as described above, and allowed to grow for two weeks to an average volume of $0.1 \mathrm{ml}$. Mice were randomly assigned into therapy groups of 4 animals and treatment started by injecting a solution of the targeted drugs or vehicle (PBS containing 1\% of DMSO) intravenously (tail vein) at the dose of $250 \mathrm{nmol} / \mathrm{Kg}$, following the schedule depicted by the arrows in the corresponding Figure. Compounds 2-5 were injected as solutions in sterile PBS containing 1\% DMSO. Animals were weighed and tumor sizes were measured daily with an electronic caliper. The tumor volume was 
calculated according to the formula (long side $) \times($ short side $) \times($ short side $) \times 0.5$. Animals were sacrificed when the termination criteria were reached. Prism 6 software (GraphPad Software) was used for data analysis (regular two-way ANOVA with the Bonferroni test).

\section{IVIS Imaging}

Female Balb/c nude mice bearing subcutaneous SKRC-52 tumors were injected intravenously with compound 6, containing the near infrared dye moiety IRDye680RD (LI-COR Biosciences) $(250 \mathrm{nmol} / \mathrm{Kg})$, dissolved in sterile PBS (100 $\mu \mathrm{l})$. Mice were anesthetized with isoflurane and fluorescence images acquired on an IVIS Spectrum imaging system (Xenogen, exposure 1s, binning factor 8, excitation at $675 \mathrm{~nm}$, emission filter at $720 \mathrm{~nm}$, f number 2, field of view 13.1). Images were taken before the injection and after $30 \mathrm{~min}, 1 \mathrm{~h}$ and $3 \mathrm{~h}$. Food and water was given ad libitum during that period.

\section{$\underline{\text { Immunofluorescence studies }}$}

SKRC-52 tumors excised from the mice after therapy study were embedded in OCT medium (Thermo Scientific), and cryostat sections $(10 \mu \mathrm{m})$ were cut. Slides were stored at $-80{ }^{\circ} \mathrm{C}$ overnight, fixed with acetone and stained using the following antibodies: mouse anti-human CAIX GT12 (Thermo Scientific), to detect the antigen, and rat anti-mouse CD31 (BD Biosciences) to detect endothelial cells. Nuclear staining was performed with DAPI. Anti-mouse IgGAlexaFluor488 (Molecular Probes by Life Technologies) and anti-rat IgG-AlexaFluor594 (Molecular Probes by Life Technologies) were then used as secondary antibodies for microscopic detection. 


\section{Results}

$\underline{\text { Synthesis and in vitro characterization of acetazolamide-based drug conjugates }}$

MMAE-dipeptide substrates, bearing a self-immolative linker and a Michael-acceptor maleimido moiety (suitable for conjugation with thiols), were synthesized in solution, as described in the Materials and Methods section and in the Supporting Information [Figure 1]. A derivative of acetazolamide (a heteroaromatic sulfonamide, capable of CAIX binding), bearing an Asp-ArgAsp-Cys tetrapeptide moiety (compound $\mathbf{1}$ in Figure 1), was then coupled to the MMAEdipeptide-maleimido derivative, yielding products $\mathbf{2 - 5}$. These compounds featured valine-alanine, valine-lysine, valine-arginine or valine-citrulline dipeptide structures as cleavable moieties, respectively, which can subsequently trigger the release of the MMAE cytotoxic moiety [Figure 1]. Compound 1 was prepared by solid phase synthesis, installing the acetazolamide moiety onto the Asp-Arg-Asp-Cys tetrapeptide linker by a copper-catalyzed azide-alkyne cycloaddition "on resin" [Figure 1].

The stability of the acetazolamide-MMAE conjugates 2-5, bearing different cleavable dipeptide sequences, was analyzed by LC/MS. The methodology detected the amounts of intact compounds at various time points, upon incubation at $37{ }^{\circ} \mathrm{C}$ in either phosphate-buffered saline (PBS) or mouse serum [Figure 2]. While no degradation of the tested conjugates was observed in PBS $\left(\mathrm{t}_{1 / 2}>96\right.$ hours in at $\left.37{ }^{\circ} \mathrm{C}\right)$, compounds $\mathbf{2 - 5}$ exhibited various degrees of chemical stability in mouse serum at $37^{\circ} \mathrm{C}$. Since free MMAE was detected as end product in LC/MS analysis, in parallel to the progressive disappearance of the conjugates, the stability profiles of Figure 2 can be related to the differential susceptibility to proteolytic cleavage of the corresponding dipeptide linkers. In particular, conjugates $\mathbf{2}$ and 5 (i.e., featuring the ValAla and ValCit linkers, respectively) showed the highest stability $\left(\mathrm{t}_{1 / 2}=23\right.$ hours and 11.2 hours), compared to the more labile compounds $\mathbf{3}$ (ValLys; $\mathrm{t}_{1 / 2}=8.2$ hours) and $\mathbf{4}\left(\right.$ ValArg; $\mathrm{t}_{1 / 2}=1.8$ hours). 


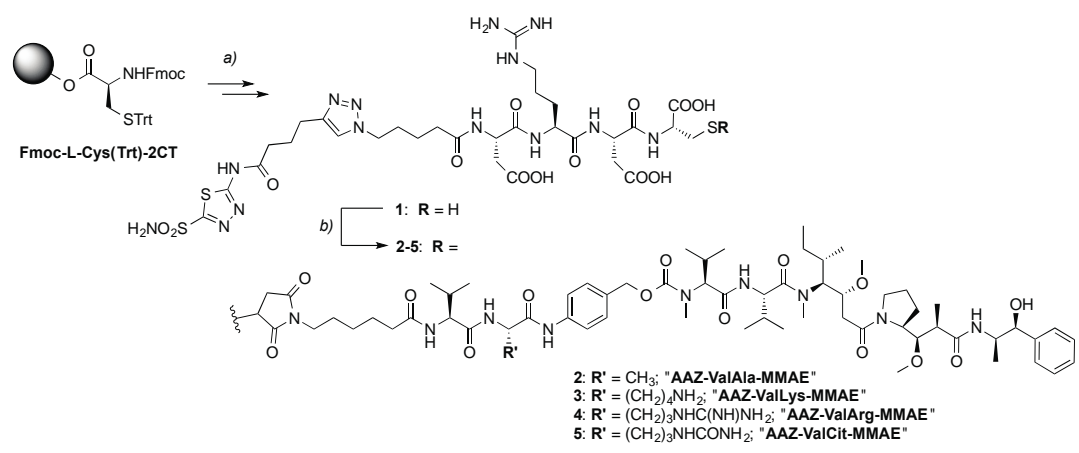

Figure 1

(2 Columns) 
A

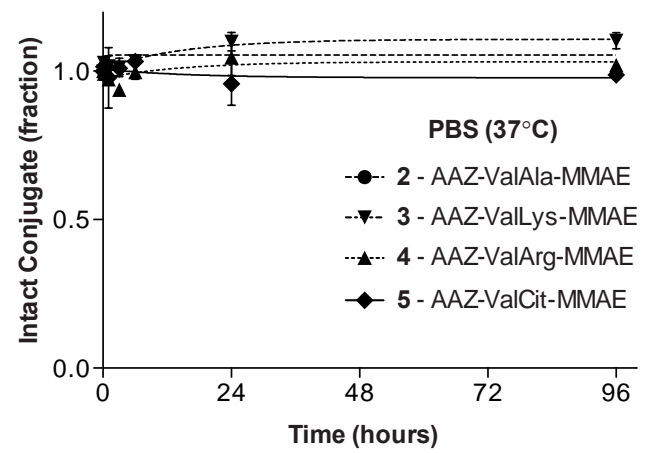

B

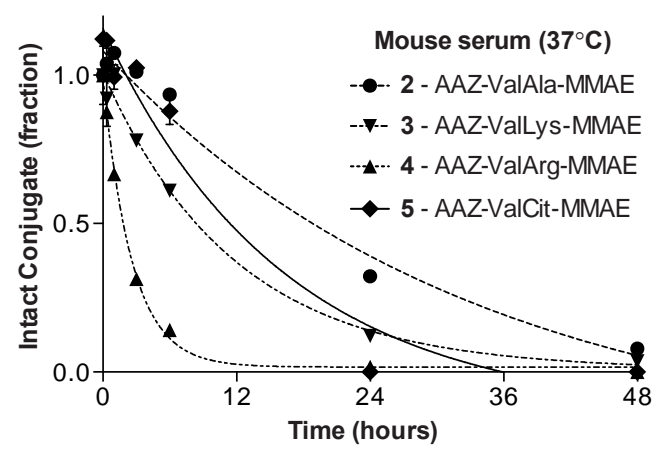

Figure 2

(1 Column) 
These data are in agreement with previous publications, $[40,41]$ which reported the lower stability of linkers featuring protonable side chains (e.g., Arg, Lys) in serum or in the presence of proteases. Considering the rapid clearance of acetazolamide derivatives from the blood (with half-lives which are typically shorter than 15 minutes) [30,33,42], the serum stability of compounds $\mathbf{2 - 5}$ is sufficient for in vivo pharmacodelivery applications, favoring a preferential tumor uptake and a slow drug release at the site of disease.

The antiproliferative activity of conjugates 2-5 was tested in vitro against CAIX-positive renal carcinoma SKRC-52 cells, using the free MMAE payload as reference compound [Figure 3]. Cells were incubated for $72 \mathrm{~h}$ in the presence of different concentrations of the tested compounds. Under this experimental procedure, the free MMAE drug was found to inhibit cell proliferation at sub-nanomolar concentrations $\left(\mathrm{IC}_{50}=0.9 \mathrm{nM}\right)$. Only a small decrease in cytotoxic activity was observed for the pro-drugs AAZ-ValLys-MMAE (3) and AAZ-ValArg-MMAE (4), which showed $\mathrm{IC}_{50}$ values of $1.6 \mathrm{nM}$ and $\mathrm{IC}_{50}=2.1 \mathrm{nM}$, respectively. By contrast, compounds AAZValAla-MMAE (2) and AAZ-ValCit-MMAE (5) were found to be substantially less potent than the free drug in vitro (i.e., $\mathrm{IC}_{50}$ values of $69 \mathrm{nM}$ and $171 \mathrm{nM}$, respectively). This observation is in keeping with the enhanced serum stability of the ValAla and ValCit linkers, compared to ValLys and ValArg [Figure 2B].

\section{Therapy experiments}

The therapeutic activity of the acetazolamide derivatives 2-5 was tested in immunodeficient Balb/c nude mice, xenografted subcutaneously with SKRC-52 [30]. Multiple intravenous injections at equivalent molar doses $(250 \mathrm{nmol} / \mathrm{Kg})$ were performed following the schedule indicated in Figure 4. All conjugates 2-5 significantly inhibited tumor growth, compared to saline treatment $(\mathrm{p}<0.0001)$ [Figure 4A]. 


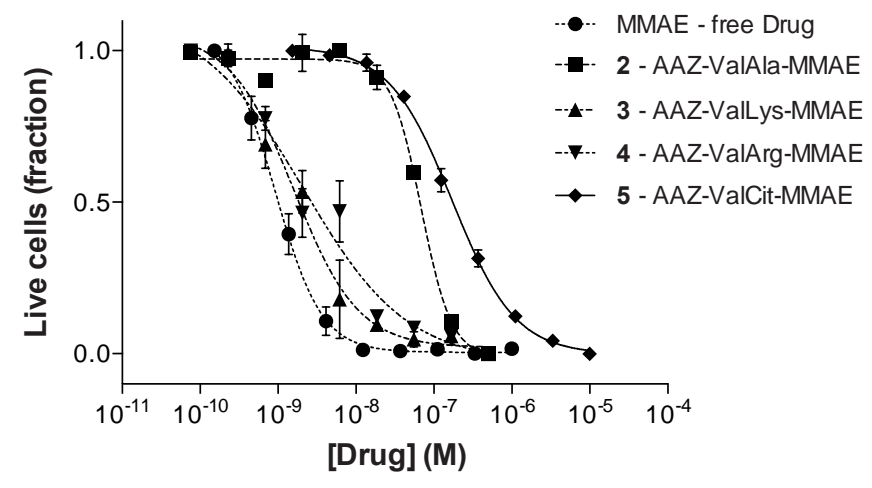

Figure 3

(1.5 Columns) 
A
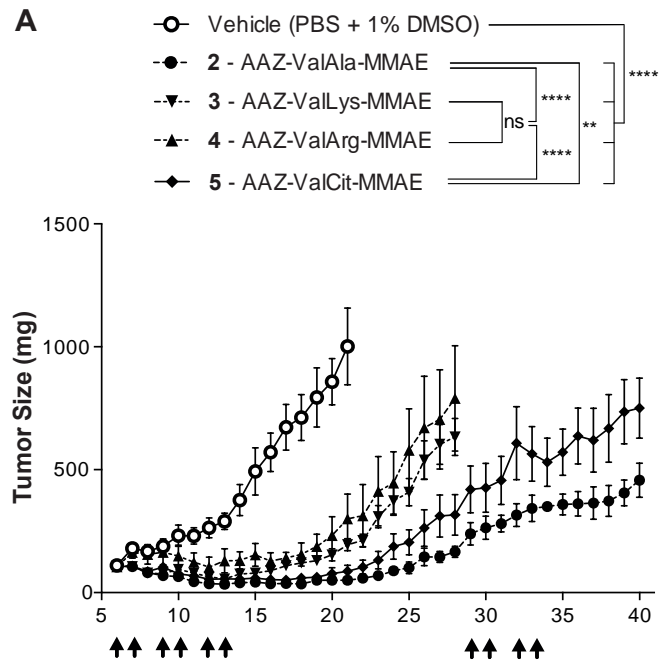

Days after tumor implantation
B -o Vehicle (PBS + 1\% DMSO)

- - 2 - AAZ-ValAla-MMAE

-7- 3 - AAZ-Vallys-MMAE

-4- 4 - AAZ-ValArg-MMAE

$\rightarrow 5$ - AAZ-ValCit-MMAE

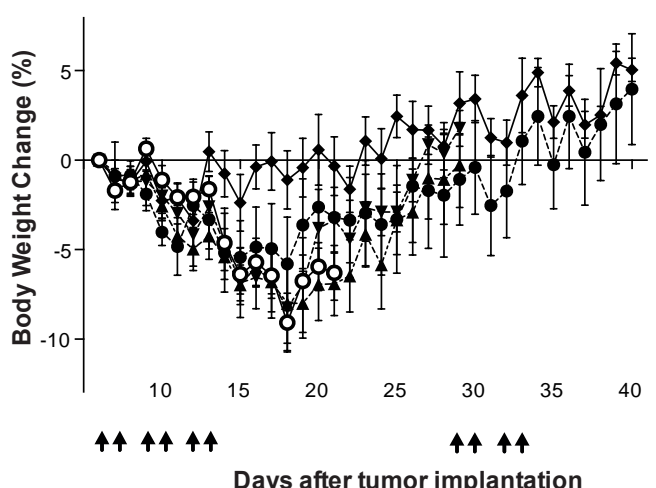

Days after tumor implantation

Figure 4

(2 Columns) 
The acetazolamide derivatives, featuring ValAla (2) and ValCit (5) as cleavable linkers, displayed the best antitumor efficacy $(\mathrm{p}<0.0001)$. None of the study groups exhibited a body weight loss of more than $10 \%$, which was comparable to the one observed in the saline treatment group [Figure 4B]. A second cycle of four injections at the same dose was administered to mice, which had enjoyed the largest tumor growth inhibition as a result of AAZ-ValAla-MMAE and AAZ-ValCitMMAE treatment. However, the second cycle of injections did not result in a tumor regression [Figure 4A]. In order to better characterize the mechanism of resistance to drug action at late time points, mice were treated with a near-infrared fluorescent derivative of acetazolamide (compound 6) [Figure 5A]. For all treatment groups, the imaging data confirmed the ability of acetazolamide to selectively target tumor lesions at the end of the therapy experiment [Figure 5B]. After sacrifice of the mice, tumor sections were stained with anti-CAIX antibodies, confirming that tumor cells had not lost antigen expression [Figure 5C]. 
A

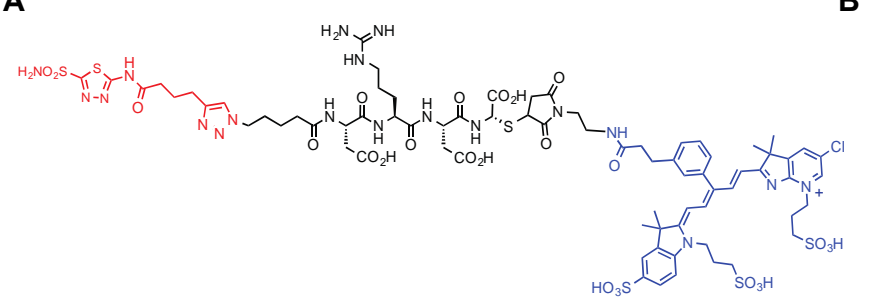

C
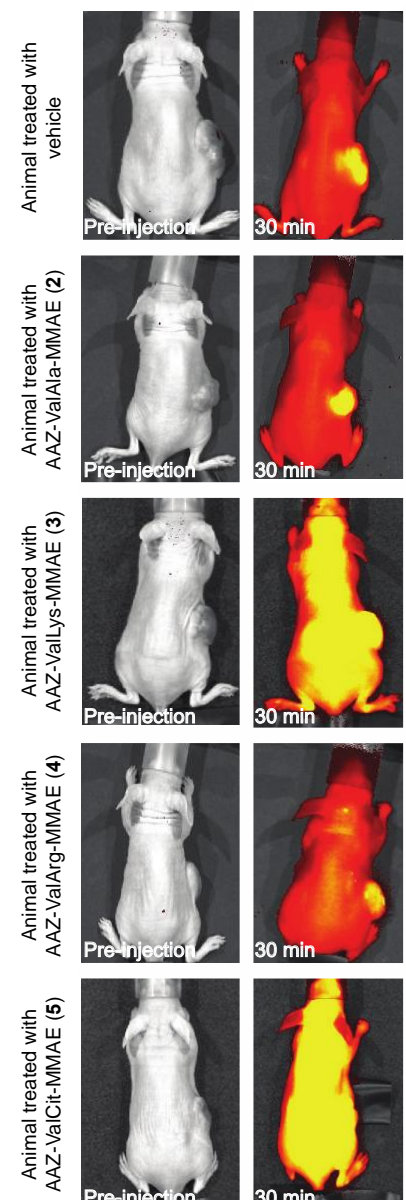

Pre-intection $30 \mathrm{~min}$

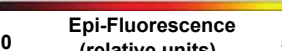

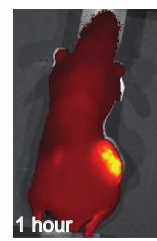
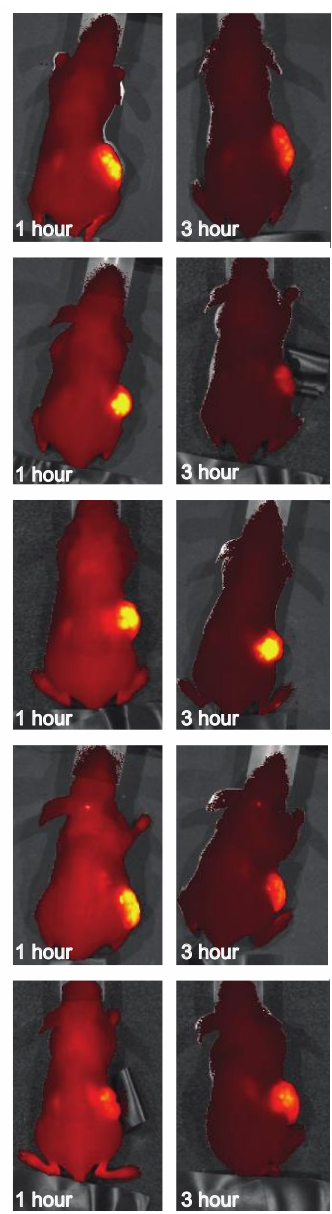

[Radiant Efficiency] $\frac{\mathrm{p} / \mathrm{s} / \mathrm{cm}^{2} / \mathrm{sr}}{\mu \mathrm{W} / \mathrm{cm}^{2}}$
B
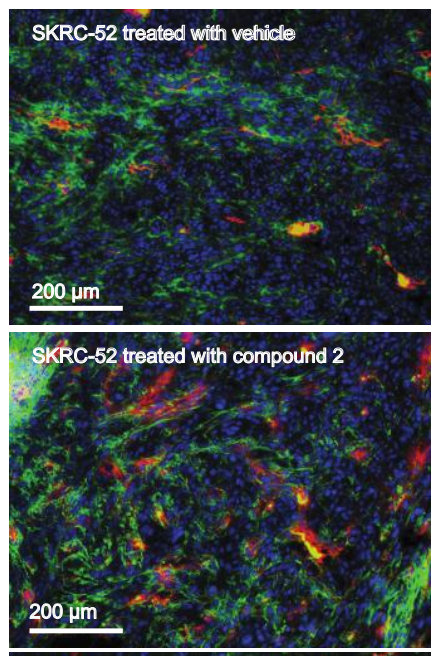

SKRC-52 treated with compound 3
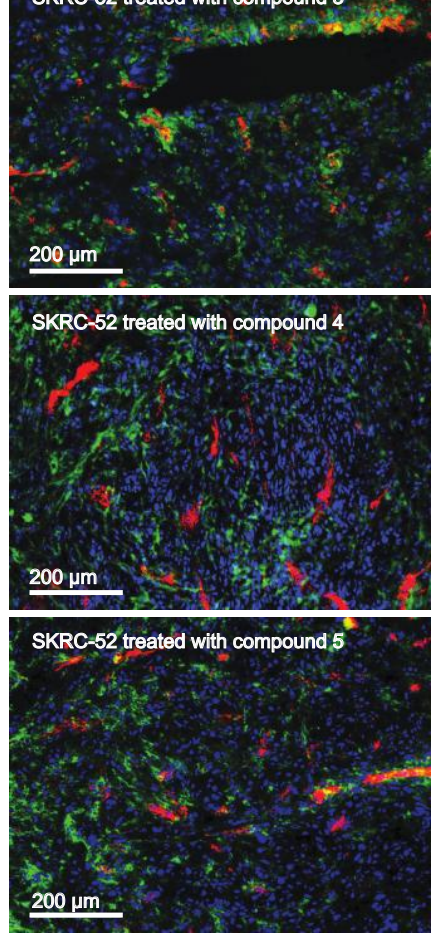

Figure 5

(2 Columns) 


\section{Discussion}

Patients with metastatic renal cell carcinoma (RCC) typically receive sunitinib or sorafenib as first-line treatment, but durable objective responses are rarely observed [43]. Both of these drugs fail to preferentially localize on tumor lesions $[44,45]$ and are poorly active in mouse models of RCC. Interestingly, sunitinib and sorafenib treatment in mice bearing SKRC52 tumors was not significantly better than saline treatment [30], while the acetazolamide derivatives $\mathbf{2}$ and $\mathbf{5}$ exhibit a substantial and prolonged tumor regression [Figure 4].

In spite of their therapeutic activity and good tolerability profile, the conjugates AAZ-ValAlaMMAE (2) and AAZ-ValCit-MMAE (5) were not able to cure mice with SKRC52 tumors, when used at the maximal tolerated dose of $250 \mathrm{nmol} / \mathrm{Kg}$. This dose is compatible with an efficient ligand localization at the tumor site, as revealed by quantitative biodistribution studies with radiolabeled acetazolamide derivatives [31,33]. The lack of sufficient therapeutic activity at later time points was not associated with loss of ligand-based targeting or of CAIX expression by tumor cells [Figure 5]. The use of human tumors grafted in nude mice does not allow to study the contribution of the adaptive arm of the immune system to tumor rejection. In the future, it will be interesting to study the therapeutic activity of compounds $\mathbf{2}$ and $\mathbf{5}$ in syngeneic tumor models, expressing CAIX. Potentially, these agents could synergize with other interventional modalities, such as external beam radiation [46] or certain immunostimulatory regimens [47] in promoting tumor rejection.

Developed in the late 90s [48], the valine-citrulline linker is one of the most broadly used dipeptide linkers in the ADC field, being included in the majority of MMAE-based ADCs in clinical studies [49], including brentuximab vedotin [50]. Interestingly, the valine-alanine dipeptide has been used in two pyrrolobenzodiazepine (PBD)-based ADCs that are currently being evaluated in 
the clinic. Notably, the CD33-targeting ADC Vadastuximab Talirine (SGN-CD33A) has recently entered pivotal phase 3 CASCADE study in patients with newly diagnosed acute myeloid leukemia (AML). For all these ADC products, the proposed mechanism of action featured a selective antibody internalization into antigen-positive cells, followed by intracellular release of the payload. We have shown, however, that both ADC linkers based on disulfides or on peptide structures can efficiently liberate drug in the tumor extracellular environment, with a therapeutic benefit in rodent models of cancer $[34,35]$.

Some examples of SMDC products, featuring protease-cleavable linkers (e.g., incorporating the ValCit [51,52] and ValAla [53,54] dipeptides) have previously been reported. Together with the uptake in healthy organs, an insufficient in vivo stability of the linker may contribute to off-target toxicities and loss of efficacy [10-12]. Our comparative evaluation of acetazolamide-peptideconjugates indicates a correlation between linker stability and activity. Acetazolamide derivatives can achieve tumor:blood ratios > 100:1 six hours after intravenous administration [31, 33]. SMDC products with a serum stability of at least few hours $\left(t_{1 / 2}>10\right.$ hours for valine-citrulline and valine-alanine derivatives) are thus able to preferentially localize at the cancer site, before releasing their payload in the tumor environment. The growing body of experimental evidence, documenting a preferential drug release in the extracellular tumor space using non-internalizing conjugates equipped with disulfide or peptidic linkers [30,31,34,35], indicates that sufficient amounts of reducing agents (e.g., glutathione) and of proteases (e.g., cathepsin B) are set free as a result of malignant cell death.

In order to facilitate a clinical translation of the best-performing MMAE conjugates, it will be essential to characterize the tumor-targeting ability of acetazolamide derivatives in patients with metastatic kidney cancer. We have recently described an acetazolamide derivative, which can be 
labeled with ${ }^{99 \mathrm{~m}} \mathrm{Tc}$ and which has recently completed GMP manufacturing [31, 33]. In full analogy to monoclonal antibodies [55,56], Nuclear Medicine studies in patients have the potential to accompany product development activities for SMDCs. 


\section{Conclusions}

Small molecule-drug conjugates, containing acetazolamide as tumor homing moiety, dipeptidebased linkers and monomethyl auristatin E as cytotoxic payload, are potently active in a mouse model of renal cell carcinoma. A comparative analysis of four dipeptide linkers (ValAla, ValLys, ValArg and ValCit) has allowed the identification of at least two acetazolamide-drug conjugates, which display sufficient stability in serum and a potent anticancer activity. The data presented in the article reinforce the concept that potent payloads can be released from non-internalizing ligands in the tumor extracellular space, when suitable linkers are used. 


\section{Acknowledgements}

Financial support from ETH Zürich, the Swiss National Science Foundation (Projects Nr. 310030B_163479/1 and SINERGIA CRSII2_160699/1), ERC Advanced Grant "Zauberkugel”, Kommission für Technologie und Innovation (Grant Nr. 17072.1), Bovena Foundation and Maiores Foundation is gratefully acknowledged. 


\section{Figure Captions}

Figure 1: Synthesis of Acetazolamide-based SMDCs (compounds 2-5). REAGENTS AND CONDITIONS: a) SPPS perfomed according to previously reported procedures [REF]; b) 1, maleimidocaproyl-Val[Cit/Ala/Arg/Lys]-MMAE, PBS/DMF 2:1, 3 h, RT, 50-90\%.

Figure 2: Comparison of the stability of $\mathbf{2 - 5}$ in PBS (A) and in mouse serum (B) at $37^{\circ} \mathrm{C}$ as determined by LC-MS. All SMDCs were found to be highly stable in saline solution $\left(t_{1 / 2}>96 \mathrm{~h}\right)$, whereas they showed lower half-lives when incubated in mouse serum. In particular, conjugates AAZ-ValArg-MMAE 4, AAZ-ValLys-MMAE 3, AAZ-ValCit-MMAE 5 and AAZ-ValAlaMMAE 2 showed crescent stability in the order (i.e. $\mathrm{t}_{1 / 2}=1.8 \mathrm{~h}, 8.2 \mathrm{~h}, 11.2 \mathrm{~h}$ and $23 \mathrm{~h}$, respectively), highlighting the effect of different linkers on biological properties.

Figure 3: Toxicity of MMAE derivatives 2-5 compared to the unmodified payload towards CAIX-expressing SKRC-52 cells. Cells were incubated for $72 \mathrm{~h}$ in the presence of crescent concentrations of the test compound at $37{ }^{\circ} \mathrm{C}$. The experiments were performed in triplicate and data points are averages values. Error bars indicate standard error of the means (SEM). Cytotoxicity values $\left(\mathrm{IC}_{50}\right)$ for AAZ-ValAla-MMAE $(2 ; 69 \mathrm{nM})$ and AAZ-ValCit-MMAE $(5 ; 171 \mathrm{nM})$ are comparable in these in vitro experiments. Both compounds demonstrate low toxicities when compared to the free payload MMAE $(0.9 \mathrm{nM})$ confirming their pro-drug behavior and the absence of efficient internalization. Conjugates AAZ-ValLys-MMAE $(3 ; 1.6 \mathrm{nM})$ and AAZValArg-MMAE $(\mathbf{4} ; 2.1 \mathrm{nM})$ show cytotoxicity comparable to MMAE $(0.9 \mathrm{nM})$, indicating possible parental free cytotoxic release during the incubation time. 
Figure 4: Therapy experiment with AAZ-dipeptide-MMAE derivatives 2-5 $(250 \mathrm{nmol} / \mathrm{Kg})$ in Balb/c nude mice bearing SKRC-52 xenografts. Graph (A) compares the therapeutic activity of the SMDCs with different dipeptide linker. In graph (B) percentage changes of body weight during the experiment are represented. The statistical analysis of the therapy result reveals superior efficacy of the acetazolamide derivatives bearing the ValAla-MMAE (2) and the ValCit-MMAE (5), when compared with AAZ-ValLys-MMAE (3) and AAZ-ValArg-MMAE (4) groups. **** indicates $\mathrm{p}<0.0001 ; * * *$ indicates $\mathrm{p}<0.001 ; * *$ indicates $\mathrm{p}<0.01$; $*$ indicates $\mathrm{p}<0.05$.

Figure 5: (A) Chemical structure of IRdye680RD fluorophore labeled with acetazolamide for the targeting of CAIX (compound 6) (B) Microscopic analysis of representative sections of SKRC-52 tumors, after treatment with acetazolamide-based SMDCs $\mathbf{2 - 5}$ or with vehicle. Green $=$ CAIX staining; Red = CD31 staining for blood vessels; Blue = DAPI staining for nuclear structures. Tumors samples from all the groups resulted to be positive for Carbonic Anhydrase IX. (C) Imaging of representative SKRC-52 bearing mice with $250 \mathrm{nmol} / \mathrm{Kg}$ of the fluorescent acetazolamide derivative 6, performed after therapy study with vehicle or with compounds 2-5. Imaging was performed after therapy study, when the animals reached termination criteria (Vehicle group: day 21; groups 3 and 4: day 28; groups 2 and 5: day 40; see also Figure 4). Selective tumor uptake of compound $\mathbf{6}$ can still be observed in all the treatment groups, as compared to the vehicle group. 


\section{References}

[1] K. Bosslet, R. Straub, M. Blumrich, J. Czech, M. Gerken, B. Sperker, H.K. Kroemer, J.P. Gesson, M. Koch, C. Monneret, Elucidation of the mechanism enabling tumor selective prodrug monotherapy, Cancer Res. 58 (1998) 1195-1201.

[2] Q. Cao, Z.-B. Li, K. Chen, Z. Wu, L. He, N. Neamati, X. Chen, Evaluation of biodistribution and anti-tumor effect of a dimeric RGD peptide-paclitaxel conjugate in mice with breast cancer, Eur. J. Nucl. Med. Mol. Imaging 35 (2008) 1489-1498.

[3] A.A.M. van der Veldt, N.H. Hendrikse, E.F. Smit, M.P.J. Mooijer, A.Y. Rijnders, W.R. Gerritsen, J.J.M. van der Hoeven, A.D. Windhorst, A.A. Lammertsma, M. Lubberink, Biodistribution and radiation dosimetry of ${ }^{11} \mathrm{C}$-labelled docetaxel in cancer patients, Eur. J. Nucl. Med. Mol. Imaging 37 (2010) 1950-1958.

[4] S.C. Alley, N.M. Okeley, P.D. Senter, Antibody-drug conjugates: targeted drug delivery for cancer, Curr. Opin. Chem. Biol. 14 (2010) 529-537.

[5] H.-P. Gerber, F.E. Koehnb, R.T. Abraham, The antibody-drug conjugate: an enabling modality for natural product-based cancer therapeutics, Nat. Prod. Rep. 30 (2013) 625-639.

[6] R.V.J. Chari, M.L. Miller, W.C. Widdison, Antibody-Drug Conjugates: An Emerging Concept in Cancer Therapy, Angew. Chem. Int. Ed. 53 (2014) 3796-3827.

[7] Y.-W. Chu, A. Polson, Antibody-drug conjugates for the treatment of B-cell non-Hodgkin's lymphoma and leukemia, Future Oncol. 9 (2013) 355-368.

[8] D.R. Mould, K.R.D. Sweeney, The pharmacokinetics and pharmacodynamics of monoclonal antibodies--mechanistic modeling applied to drug development, Curr. Opin. Drug Discov. Devel. 10 (2007) 84-96.

[9] C.V. Pastuskovas, E.E. Mundo, S.P. Williams, T.K. Nayak, J. Ho, S. Ulufatu, S. Clark, S. Ross, E. Cheng, K. Parsons-Reponte, G. Cain, M. Van Hoy, N. Majidy, S. Bheddah, J. dela 
Cruz Chuh, K.R. Kozak, N. Lewin-Koh, P. Nauka, D. Bumbaca, M. Sliwkowski, J. Tibbitts, F.-P. Theil, P.J. Fielder, L.A. Khawli, C.A. Boswell, Effects of anti-VEGF on pharmacokinetics, biodistribution, and ttumor penetration of trastuzumab in a preclinical breast cancer model, Mol. Cancer Ther. 11 (2012) 752-762.

[10] H. Donaghy, Effects of antibody, drug and linker on the preclinical and clinical toxicities of antibody-drug conjugates, mAbs 8 (2016) 659-671

[11] E. R. Boghaert, K. M. Khandke, L. Sridharan, M. Dougher, J. F. DiJoseph, A. Kunz, P. R. Hamann, J. Moran, I. Chaudhary, N. K. Damle, Determination of pharmacokinetic values of calicheamicin-antibody conjugates in mice by plasmon resonance analysis of small (5 microl) blood samples, Cancer Chemother. Pharmacol. 61 (2008) 1027-1035.

[12] C. Wei, G. Zhang, T. Clark, F. Barletta, L. N. Tumey, B. Rago, S. Hansel, X. Han, Where Did the Linker-Payload Go? A Quantitative Investigation on the Destination of the ReleasedLinker-Payload from an Antibody-Drug Conjugate with a Maleimide Linker in Plasma, Anal Chem. 88 (2016) 4979-486.

[13] G. Casi, D. Neri, Antibody-drug conjugates and small molecule-drug conjugates: opportunities and challenges for the development of selective anticancer cytotoxic agents, J. Med. Chem. 58 (2015) 8751-8761.

[14] M.S. Dennis, H.K. Jin, D. Dugger, R.H. Yang, L. McFarland, A. Ogasawara, S. Williams, M.J. Cole, S. Ross, R. Schwall, Imaging tumors with an albumin-binding Fab, a novel tumortargeting agent, Cancer Res. 67 (2007) 254- 261.

[15] E. Vlashi, J.E. Sturgis, M. Thomas, P.S. Low, Real time, noninvasive imaging and quantitation of the accumulation of ligand-targeted drugs into receptor-expressing solid tumors, Mol. Pharm. 6 (2009) 1868-1875. 
[16] S.A. Kularatne, K. Wang, H.K.R. Santhapuram, P.S. Low, Prostate-specific membrane antigen targeted imaging and therapy of prostate cancer using a PSMA inhibitor as a homing ligand, Mol. Pharm. 6 (2009) 780-789.

[17] W. Xia, P.S. Low, Folate-targeted therapies for cancer, J. Med. Chem. 53 (2010) 6811-6824.

[18] I.R. Vlahov, C.P. Leamon, Engineering folate-drug conjugates to target cancer: from chemistry to clinic, Bioconjugate Chem. 23 (2012) 1357-1369.

[19] S.A. Kularatne, C. Venkatesh, H.-K.R. Santhapuram, K. Wang, B. Vaitilingam, W.A. Henne, P.S. Low, Synthesis and biological analysis of prostate-specific membrane antigen-targeted anticancer prodrugs, J. Med. Chem. 53 (2010) 7767-7777.

[20] J.B. Engel, A.V. Schally, J. Dietl, L. Rieger, A. Hönig, Targeted therapy of breast and gynecological cancers with cytotoxic analogues of peptide hormones, Mol. Pharm. 4 (2007) 652658.

[21] M. Srinivasarao, C.V. Galliford, P.S. Low, Principles in the design of ligand-targeted cancer therapeutics and imaging agents, Nat. Rev. Drug Discov. 14 (2015) 203-219.

[22] A. Thiry, J.M. Dogne, B. Masereel, C.T. Supuran, Targeting tumor-associated carbonic anhydrase IX in cancer therapy, Trends Pharmacol. Sci. 27 (2006) 566-573.

[23] S. Pastorekova, J. Zavada, Carbonic anhydrase IX (CA IX) as a potential target for cancer therapy, Cancer Ther. 2 (2004) 245-262.

[24] C. T. Supuran, Inhibition of carbonic anhydrase IX as a novel anticancer mechanism. World J. Clin Oncol. 7 (2012), 98-103.

[25] C.C. Wykoff, N.J. Beasley, P.H. Watson, K.J. Turner, J. Pastorek, A. Sibtain, G.D. Wilson, H. Turley, K.L. Talks, P.H. Maxwell, C.W. Pugh, P.J. Ratcliffe, A.L. Harris, Hypoxiainducible expression of tumor-associated carbonic anhydrases, Cancer Res. 60 (2000) 70757083. 
[26] F.G. van Schaijk, E. Oosterwijk, J.D. Molkenboer-Kuenen, A.C. Soede, B.J. McBride, D.M. Goldenberg, W.J. Oyen, F.H. Corstens, O.C. Boerman, Pretargeting with bispecific anti-renal cell carcinoma x anti-DTPA(In) antibody in 3 RCC models, J. Nucl. Med. 46 (2005) 495-501.

[27] P.-C. Lv, K.S. Putt, P.S. Low, Evaluation of Nonpeptidic Ligand Conjugates for SPECT Imaging of Hypoxic and Carbonic Anhydrase IX-Expressing Cancers, Bioconjugate Chem. 27 (2016) 1762-1769.

[28] M.C. Lloyd, J.J. Cunningham, M.M. Bui, R.J. Gillies, J.S. Brown, R.A. Gatenby, Darwinian dynamics of intratumoral heterogeneity: not solely random mutations but also variable environmental selection forces, Cancer Res. 76 (2016) 3136-3144.

[29] H.M. Petrul, C.A. Schatz, C.C. Kopitz, L. Adnane, T.J. McCabe, P. Trail, S. Ha, Y.S. Chang, A. Voznesensky, G. Ranges, P.P. Tamburini, Therapeutic mechanism and efficacy of the antibody-drug conjugate BAY 79-4620 targeting human carbonic anhydrase 9, Mol. Cancer Ther. 11 (2012) 340-349.

[30] N. Krall, F. Pretto, W. Decurtins, G.J.L. Bernardes, C.T. Supuran, D. Neri, A SmallMolecule Drug Conjugate for the Treatment of Carbonic Anhydrase IX Expressing Tumors, Angew. Chem. Int. Ed. 53 (2014) 4231-4235.

[31] S. Cazzamalli, A. Dal Corso, D. Neri, Acetazolamide serves as selective delivery vehicle for dipeptide-linked drugs to renal cell carcinoma, Mol. Cancer Ther. (2016), http://dx.doi.org/10.1158/1535-7163.MCT-16-0283.

[32] N. Krall, F. Pretto, D. Neri, A bivalent small molecule-drug conjugate directed against carbonic anhydrase IX can elicit complete tumour regression in mice, Chem. Sci. 5 (2014) 36403644.

[33] N. Krall, F. Pretto, M. Mattarella, C. Müller, D. Neri, A 99mTc-labeled ligand of carbonic anhydrase IX selectively targets renal cell carcinoma in vivo, J. Nucl. Med. 57 (2016) 943-949. 
[34] E. Perrino, M. Steiner, N. Krall, G.J.L. Bernardes, F. Pretto, G. Casi, D. Neri, Curative properties of noninternalizing antibody-drug conjugates based on maytansinoids, Cancer Res. 74 (2014) 2569-2578.

[35] R. Gébleux, S. Wulhfard, G. Casi, D. Neri, Antibody format and drug release rate determine the therapeutic activity of noninternalizing antibody-drug conjugates, Mol. Cancer Ther. 14 (2015) 2606-2612.

[36] L. Brülisauer, M.A. Gauthier, J.-C. Leroux, Disulfide-containing parenteral delivery systems and their redox-biological fate, J. Control. Release 195 (2014) 147-154.

[37] N.H. Bander, Antibody-drug conjugate target selection: critical factors, Methods Mol. Biol. 1045 (2013) 29-40.

[38] S. Xu, Internalization, trafficking, intracellular processing and actions of antibody-drug conjugates, Pharm. Res. 32 (2015) 3577-3583.

[39] B. Yoo, K. Ma, L. Zhang, A. Burns, S. Sequeira, I. Mellinghoff, C. Brennan, U. Wiesner, M.S. Bradbury, Ultrasmall dual-modality silica nanoparticle drug conjugates: design, synthesis, and characterization, Bioorg. Med. Chem. 15 (2015) 7119-7130.

[40] S.O. Doronina, B.E. Toki, M.Y. Torgov, B.A. Mendelsohn, C.G. Cerveny, D.F. Chace, R.L. DeBlanc, R.P. Gearing, T.D. Bovee, C.B. Siegall, J.A. Francisco, A.F. Wahl, D.L. Meyer, P.D. Senter, Development of potent monoclonal antibody auristatin conjugates for cancer therapy, Nat. Biotechnol. 21 (2003) 778-784.

[41] G.M. Dubowchik, R.A. Firestone, L. Padilla, D. Willner, S.J. Hofstead, K. Mosure, J.O. Knipe, S.J. Lasch, P.A. Trail, Cathepsin B-labile dipeptide linkers for lysosomal release of doxorubicin from internalizing immunoconjugates: model studies of enzymatic drug release and antigen-specific in vitro anticancer activity, Bioconjugate Chem. 13 (2002) 855-869. 
[42] M. Wichert, N. Krall, W. Decurtins, R.M. Franzini, F. Pretto, P. Schneider, D. Neri, J. Scheuermann, Dual-display of small molecules enables the discovery of ligand pairs and facilitates affinity maturation. Nature Chem. 7 (2015) 241-249.

[43] S. Fischer, S. Gillessen, C. Rothermundt, Sequence of treatment in locally advanced and metastatic renal cell carcinoma, Transl. Androl. Urol. 4 (2015) 310-325.

[44] M. Kuchar, M.C. Oliveira, L. Gano, I. Santos, T. Kniess, Radioiodinated sunitinib as a potential radiotracer for imaging angiogenesis-radiosynthesis and first radiopharmacological evaluation of 5-[25 I]Iodo-sunitinib, Bioorg. Med. Chem. Lett. 22 (2012) 2850-2855.

[45] A.J. Poot, B. van der Wildt, M. Stigter-van Walsum, M. Rongen, R.C. Schuit, N.H. Hendrikse, J. Eriksson, G.A.M.S. van Dongen, A.D. Windhorst, $\left[{ }^{11} \mathrm{C}\right]$ Sorafenib: radiosynthesis and preclinical evaluation in tumor-bearing mice of a new TKI-PET tracer, Nucl. Med. Biol. 40 (2013) 488-497.

[46] G.S. Higgins, S.M. O'Cathail, R.J. Muschel, W.G. McKenna, Drug radiotherapy combinations: review of previous failures and reasons for future optimism, Cancer Treat. Rev. 41 (2015) 105-113.

[47] H.-P. Gerber, P. Sapra, F. Loganzo, C. May, Combining antibody-drug conjugates and immune-mediated cancer therapy: What to expect?, Biochem. Pharmacol. 102 (2016) 1-6.

[48] G.M. Dubowchik, R.A. Firestone, Cathepsin B-sensitive dipeptide prodrugs. 1. A model study of structural requirements for efficient release of doxorubicin, Bioorg. Med. Chem. Lett. 8 (1998) 3341-3346.

[49] N. Jain, S.W. Smith, S. Ghone, B. Tomczuk, Current ADC linker chemistry, Pharm. Res. 32, (2015) 3526-3540. 
[50] P.D. Senter, E.L. Sievers, The discovery and development of brentuximab vedotin for use in relapsed Hodgkin lymphoma and systemic anaplastic large cell lymphoma, Nat. Biotechnol. 30 (2012) 631-637.

[51] J.L. Crisp, E.N. Savariar, H.L. Glasgow, L.G. Ellies, M.A. Whitney, R.Y. Tsien, Dual targeting of integrin $\alpha v \beta 3$ and matrix metalloproteinase- 2 for optical imaging of tumors and chemotherapeutic delivery, Mol. Cancer. Ther. 13 (2014) 1514-1525.

[52] A. Dal Pozzo, E. Esposito, M. Ni, L. Muzi, C. Pisano, F. Bucci, L. Vesci, M. Castorina, S. Penco. Conjugates of a novel 7-substituted camptothecin with RGD-peptides as $\alpha v \beta 3$ integrin ligands: an approach to tumor-targeted therapy, Bioconjugate Chem. 21 (2010) 1956-1967.

[53] K. Hochdörffer, K. Abu Ajaj, C. Schäfer-Obodozie, F. Kratz, Development of novel bisphosphonate prodrugs of doxorubicin for targeting bone metastases that are cleaved $\mathrm{pH}$ dependently or by cathepsin B: synthesis, cleavage properties, and binding properties to hydroxyapatite as well as bone matrix, J. Med. Chem. 55 (2012) 7502-7515.

[54] A. Dal Corso, M. Caruso, L. Belvisi, D. Arosio, U. Piarulli, C. Albanese, F. Gasparri, A. Marsiglio, F. Sola, S. Troiani, B. Valsasina, L. Pignataro, D. Donati, C. Gennari, Synthesis and biological evaluation of RGD peptidomimetic-paclitaxel conjugates bearing lysosomally cleavable linkers, Chem. Eur. J. 21 (2015) 6921-6929.

[55] G.L. Poli, C. Bianchi, G. Virotta, A. Bettini, R. Moretti, E. Trachsel, G. Elia, L. Giovannoni, D. Neri, A. Bruno, Radretumab radioimmunotherapy in patients with brain metastasis: a 124IL19SIP dosimetric PET study, Cancer Immunol. Res. 1 (2013) 134-143.

[56] D.A. Heuveling, R. de Bree, D.J. Vugts, M.C. Huisman, L. Giovannoni, O.S. Hoekstra, C.R. Leemans, D. Neri, G.A.M.S. van Dongen, Phase 0 microdosing PET study using the human mini antibody F16SIP in head and neck cancer patients, J. Nucl. Med. 54 (2013) 397-401. 
Non internalizing Small Molecule-Drug Conjugates

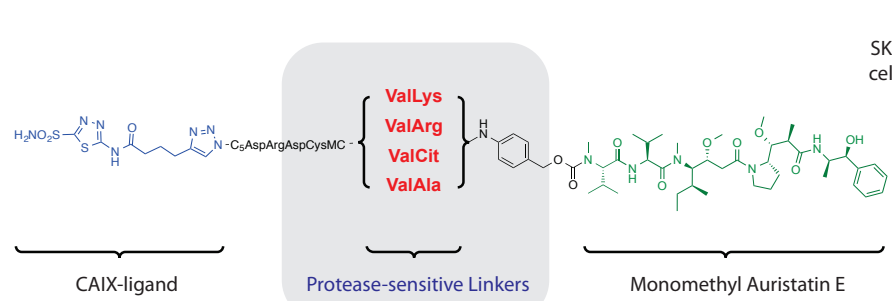

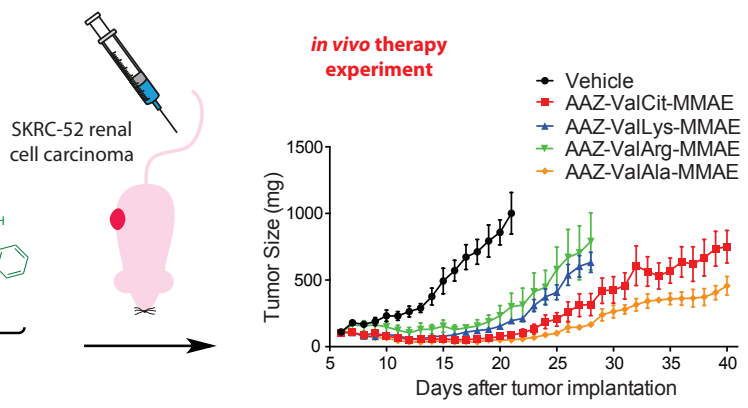

